\title{
DUALITY AND REGGE ABSORPTION MODELS
}

\author{
R.P. WORDEN* \\ Physics Department, University of Michigan, Ann Arbor, Michigan
}

Received 19 February 1973

\begin{abstract}
Pion-nucleon charge-exchange FESRs show that the best current Regge absorption models are not dual. Their strong disagreement with the FESRs is surprising, in view of the well-known correspondence between absorptive zeroes and low-energy resonance zeroes. We show that the non-duality does not arise from small details of the models, but follows inevitably from certain basic features which they share. To give the absorption the required phase, current models all introduce a strong low-lying $j$-plane component in the region $0>j>-1$. This component over whelms the $\rho$ pole at low energies, producing a non-dual amplitude. Some possible remedies are discussed.
\end{abstract}

\section{Introduction}

Duality has been one of the most fruitful ideas to emerge from two-body Regge phenomenology, and has found applications in all areas of strong-interaction physics. However, duality was proposed at a time when Regge poles alone were thought to give an adequate description of high-energy amplitudes. It has since become clear that Regge cuts - in particular, absorptive corrections - must play an important role [1]. For instance $K^{ \pm} p$ and $p^{ \pm} p$ elastic crossovers show [2] that imaginary parts of non-flip $\omega$-exchange amplitudes have a peripheral, absorbed $t$-dependence. Similarly the sharp forward peak in $\pi$-exchange processes is most simply described by a strong absorption model $[2,11]$.

It is therefore important to extend the idea of duality to include absorptive cuts as well as Regge poles.

There is a well-known qualitative consistency between absorption models and duality [3]. Absorption of Regge poles produces a high-energy amplitude in which only the peripheral partial waves are large; in low-energy phase-shift analyses the prominent resonances occur in the same peripheral band of partial waves, at impact parameters around $1 \mathrm{fm}$. This similarity of low-energy and high-energy amplitudes is reflected in the fixed- $t$ zero structure, which persists from low to high energies. Such "qualitative duality" is very encouraging, and suggests that duality can indeed

* Now at Rutherford High Energy Laboratory, Chilton, Didcot, Berkshire, England. 
be extended to include realistic high-energy amplitudes containing strong absorptive cuts. However, it is only a beginning; one would like to make duality more quantitative, so as to constrain sizes of amplitudes, as well as their zero structure. More important, recent amplitude analyses [4,5] have shown that high-energy amplitudes are more complicated than the simplest absorption picture suggests. For instance, imaginary parts of tensor-exchange amplitudes probably do not have an absorptive zero structure [5]; one would like to know how this feature is dual to low-energy amplitudes. New, more refined Regge absorption models have been proposed [6-10], which can describe the amplitude analysis results. They are certainly more realistic than the original absorption models, and may represent progress towards a good description of all high energy two-body amplitudes. It is therefore important to ask whether these new absorption models are dual.

This paper is an attempt to answer that question, by studying the pion-nucleon charge-exchange amplitudes. At high energies, the new absorption models have been fitted to the $\pi^{-} \mathrm{p} \rightarrow \pi^{0} \mathrm{n}$ amplitudes; they can be analytically continued to low energies and compared with a local average of the phase-shift amplitudes. The comparison is made through finite energy sum rules.

In sect. 2 we clarify our usage of the word "duality", and explain how the FESRs are used as tests of the duality of high energy models. Readers primarily interested in results may skip this section.

Sect. 3 contains FESR comparisons of three Regge absorption models. The original strong absorption model [11] shows the correct qualitative features of the FESRs; however, it gives the wrong overall magnitude in the non-flip FESR. Next, we examine two representative new versions of the absorption model - the HartleyKane model [6] and the Ringland et al. " $i$-factor" model [7]. These models, though differing in theoretical motivation, can both fit the high-energy $\pi^{-} p \rightarrow \pi^{0} \mathrm{n}$ amplitudes (unlike the old strong cut model). However, they both give essentially the same clear disagreement with FESR duality. The helicity non-flip FESR should have a zero, at $t \simeq-0.1$, which corresponds to the peripheral zero in the high-energy amplitude. Neither model gives this FESR zero; furthermore, they give an incorrect FESR sign at $t=0$. I have made the same FESR comparisons for two other new absorption models (a typical Regge-Regge cut model [8], and the Girardi et al. "RPR" model [9]); these give the same unacceptable FESR result.

This is the main result of the paper; that the best current forms of the Regge absorption model are not dual. The clear-cut FESR disagreement, and the similarity of the different models, are perhaps encouraging. They suggest that it is not a matter of certain models being wrong, or of parameters needing adjustment, but that there is a simple underlying cause of non-duality, common to all new absorption models, from which we may learn something.

In sect. 4 we show that the duality problem arises through analyticity. All the new models alter the phase of the absorptive correction (relative to the old strong absorption model), to agree with amplitude analysis results. In modifying the phase of the absorption, one naturally modifies its energy dependence; this altered energy- 
dependence conflicts with local-average and FESR duality. These effects are most clearly seen in terms of the $j$-plane structure of the models.

The conflict between absorption and duality does not imply that one should abandon present forms of the absorption model; rather, it suggests a clear direction in which one should try to improve them. In sect. 5 we discuss a variety of possibilities for this improvement, and some tests of their predictions.

\section{Phenomenological duality and FESR's}

Duality is based on the empirical fact that certain simple features of high-energy amplitudes persist, in a local average sense, down to low energies [12]. Typical "simple features" are a fixed- $t$ zero structure, reality of the amplitude, or an approximately power-law energy dependence. Thus an amplitude is dual if its high energy approximation, when extrapolated down, gives a local average of the actual amplitude at low and intermediate energies*. The word "approximation" is crucial; the exact high-energy amplitude extrapolates down to give the exact low energy amplitude, so cannot be used to define duality. One must make the meaning of "approximation" precise, since different approximations can be very similar at high energies but extrapolate differently to the low-energy region.

In Regge-pole models, the approximation was that of including only a few leading Regge poles, and neglecting all other (low-lying) contributions to the amplitude. A Regge pole contribution was written in the form $(s-u)^{\alpha}$ (with no daughter terms $\left.(s-u)^{\alpha-n}\right)$, and so had a well-defined continuation to low energies, making duality a well-defined statement. Now, however, we know that absorptive corrections are important $[1,2]$; these give in general a $j$-plane cut, which may have daughter cuts, depending on how the absorption is calculated. In this case, too, for tests of duality the high-energy approximation must be uniquely defined. I shall define it in terms of $j$-plane structure**.

The leading singularities are taken to be all poles or regions of cut within a distance $\Delta j$ of the highest-lying $j$-plane singularity. The rest will be called the non-leading part. Initially, for definiteness, I shall take $\Delta j=1.0$. Then the high-energy approximation is an amplitude which contains only the leading poles (and cut regions) of the actual amplitude. Duality implies that this high-energy approximation localaverages the actual amplitude at low-intermediate energies. In other words, in the local average of the amplitude, non-leading contributions are small.

* The phrases "low and intermediate" and "local average" can easily be quantified. For twobody amplitudes one could take low and intermediate to mean $p_{\mathrm{lab}}>0.5 \mathrm{GeV} / \mathrm{c}$ and local average to mean average in the imaginary part over an interval $\Delta p_{\text {lab }} \simeq 1 \mathrm{GeV} / c$. One could similarly define how accurate the local average is supposed to be. Resonance saturation of imaginary parts of amplitudes is a further, separate assumption.

** More precisely, in terms of Khuri plane structure. For the rest of this paper, "single pole behaviour" will mean single Khuri pole behaviour $(s-u)^{\alpha}$, rather than single Regge pole behaviour, $P^{\alpha}\left(\cos \theta_{t}\right)$, and "the $j$-plane" will be taken to mean strictly "the Khuri plane". 
How small is "small"? Different Regge representations (for instance $s^{\alpha}, \frac{1}{2}(s-u)^{\alpha}$, $\left.\left(s-m^{2}\right)^{\alpha}\right)$ give different definitions of daughters; an amplitude without daughters in one representation has daughters in another. Presumably the non-leading singularities cannot be much smaller than these daughters. Duality will be taken to mean that they are not much larger, either. The daughters in a one-term Veneziano model are small in this sense [13].

This definition of a dual amplitude accords with accepted ideas of duality [12], and is a non-trivial, testable statement (i.e. it is possible that some amplitudes of the real world are not dual in this sense). Other definitions are possible, perhaps not involving the idea that low-lying singularities are small. However, if low-lying effects are large, they cannot be arbitrary; otherwise the continuation from high to low energies is arbitrary, and duality is undefinable.

Finite energy sum rules [12] are based on the fixed- $t$ contour integral shown in fig. 1 . They are always written for amplitudes $T(\nu)$ which are odd in the crossingsymmetric energy variable, $\nu=\frac{1}{2}(s-u)$, so that the two "semi-circle" contributions $\int_{b}^{c}$ and $\int_{d}^{a}$ are equal (with the same sign); the two real-axis contributions are also equal. Cauchy's theorem then implies the FESR:

$$
\int_{a}^{b} T(\nu) \mathrm{d} \nu=-\int_{b}^{c} T(\nu) \mathrm{d} \nu .
$$

The left-hand side of this equation is calculable from phase-shift amplitudes. To test a high-energy model, one evaluates the right-hand side (the integration round the semicircle) from the model amplitudes. Duality implies that the non-leading $j$-plane contributions to the model amplitude are small in the local average at intermediate energies, and therefore small in the semi-circle integral. This is the crucial assumption which makes the FESRs usable - only the leading singularities are needed in the evaluation of the semi-circle integral. Then if eq. (1) is not roughly satisfied as a function of momentum transfer $t$, the Regge model is not dual.

In a Regge-pole model, the semicircle integral can be done analytically; a pole of the form $(s-u)^{\alpha}$ gives an integral

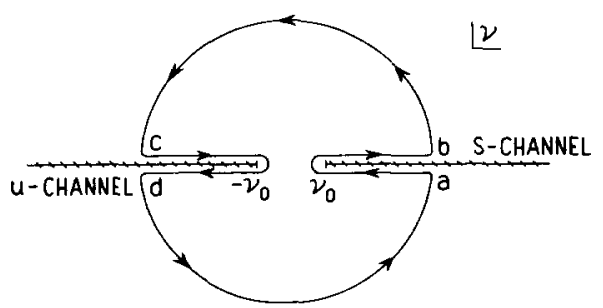

Fig. 1. The contour of integration used in defining the FESR. The energy variable is $\nu=\frac{1}{2}(s-u)$ and the integration is done at fixed momentum transfer, $t$. The integrals over physical region amplitudes (e.g. $f_{a}^{b}$ ) include any sub-threshold poles. 
$\sim \frac{\sin \frac{1}{2} \pi(\alpha+1)}{(\alpha+1)} N^{\alpha+1}$

However, if the high-energy model contains $j$-plane cuts, it is simplest to evaluate the semi-circle integral numerically. For this purpose it is important to write the cut amplitude in a form which is explicitly Hermitian-analytic and odd in $\nu$. One must also use a form with no daughter poles or cuts, to minimise the effect of non-leading contributions on the FESR.

\section{FESR tests of absorption models}

This section describes the FESR comparison* of three typical Regge absorption models in $\pi^{-} \mathrm{p} \rightarrow \pi^{0} \mathrm{n}$. The first is a basic strong absorption model [11], of the old variety which do not fit recent amplitude analysis results. The other two are typical of the new generation of models, which can describe amplitude analysis results and data in a wide variety of reactions (they are not just models of $\pi^{-} p \rightarrow \pi^{0} n$ ). These are the Hartley-Kane absorption model [6] and the Ringland et al. " $i$-factor" model [7]. I shall first briefly describe all the models, emphasising their amplitude structure rather than their motivation, and then discuss their FESR comparisons, which are shown on figs. 2-4. Details of amplitude notation and FESRs are given in appendix 1. Appendix 2 contains the numerical details of the models, including the ways of calculating the amplitudes in an exactly crossing symmetric, daughterless form.

\subsection{The basic strong absorption model**}

The absorption model is most simply motivated by a double-scattering picture, in which the absorption correction represents reggeon exchange preceded (or followed) by elastic scattering. In the basic absorption model, the elastic scattering amplitude is approximated by a pure imaginary pomeron pole exchange term:

$$
T_{\mathrm{el}}=i s \sigma_{\infty} \exp \left[\frac{1}{2} a t\right] \text {. }
$$

The absorptive correction is obtained by a two-dimensional convolution of this amplitude with the $\rho$ pole amplitude. This gives a $j$-plane cut with fixed branch point $\alpha_{c}=\alpha_{\rho}(0)$, which interferes more or less destructively with the $\rho$ pole amplitude. The cut must be strong enough to produce a crossover zero at $t \simeq-0.2$ in the imaginary part of the non-flip amplitude, and to give a dip in the cross section at $t \simeq-0.6$ by destruc tive pole-cut interference in the spin-flip amplitude. To do this, the non-flip and spinflip euts are each multiplied by phenomenological " $\lambda$ factors" of order $1.5-2$.

This model gives strong absorption (and therefore "crossover" zeroes at small $t$ )

\footnotetext{
* Some cut models have also been compared with $\pi^{-} p \rightarrow \pi^{0} n$ FESRs by Johnson [14] and Michael [32].

** See ref. [11].
} 

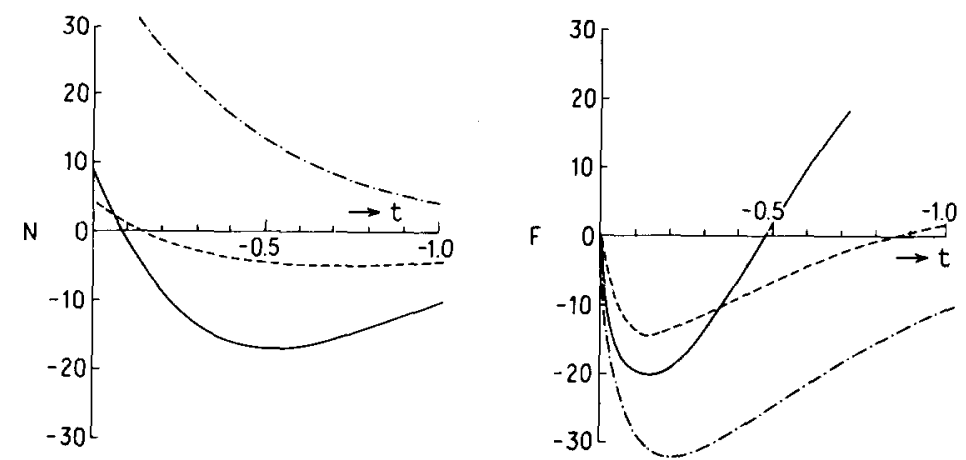

Fig. 2. Comparison of left- and right-hand sides of the FESR (eq. (1)) as a function of momentum transfer. The solid curve is the phase-shift integral, and the dashed curve is the semicircle integral evaluated in the basic strong cut model. The dash-dot curve is the $\rho$ pole contribution to the integral. $N$ is the "asymptotic" $s$-channel non-flip amplitude $\sim\left(A^{-}+\omega B^{-}\right)$, and $F$ is the "asymptotic" s-channel flip amplitude $\sim \sqrt{-t} A^{-}$.
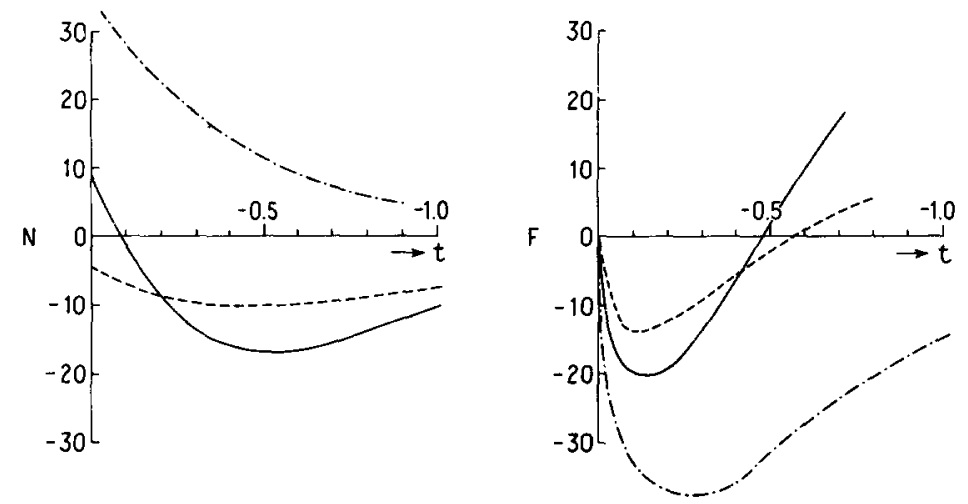

Fig. 3. As for fig. 2, except that the dashed curve is the semicircle integral evaluated in the Hartley-Kane model. The dash-dot curve is the $\rho$ pole contribution in this model.
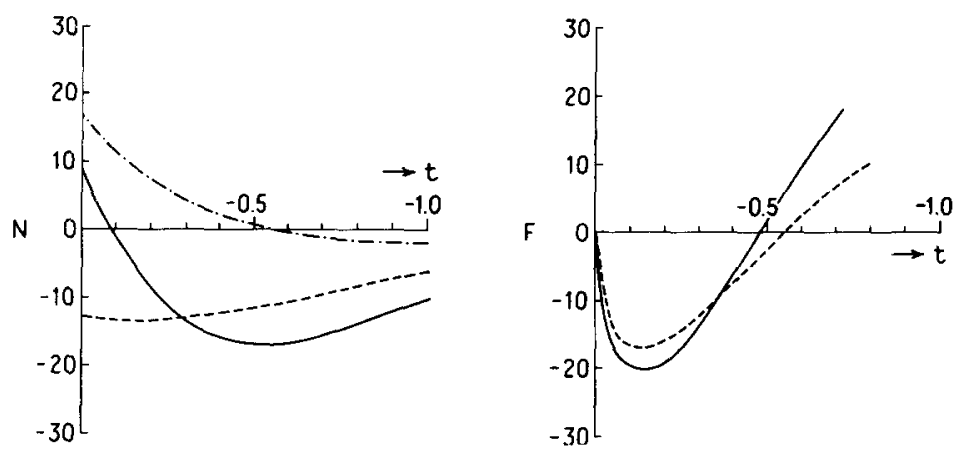

Fig. 4. As for fig. 2, except that the dashed curve is the semicircle integral evaluated in the Ringland et al. $i$-factor model. The dash-dot curve is the $\rho$ pole contribution in this model (with a wrong-signature zero at $\alpha_{\rho}=0$ ). 
in both real and imaginary parts of the non-flip amplitude. However, amplitude analysis [4] shows that the real part of this amplitude probably does not have a zero at small $t$, so the real part is not strongly absorbed. In the other two models the phase of the absorptive correction is altered, in order to give strong absorption of imaginary parts and weak absorption of real parts of vector-exchange amplitudes.

\subsection{The Hartley-Kane absorption model*}

Hartley and Kane have proposed an absorption model in which the elastic scattering amplitude $T_{\mathrm{el}}$ (eq. (2)) is replaced by a two-component effective elastic amplitude $T_{\text {eff }}$, which is approximately of the form:

$$
\left.T_{\text {eff }}=i \nu\left[a \mathrm{e}^{b t}+c \mathrm{e}^{d t} J_{0}\left(R_{0} \sqrt{-t\left[\ln \nu-\frac{1}{2} i \pi\right.}\right]\right)\right]
$$

$T_{\text {eff }}$ represents the effect of elastic and diffractively produced intermediate states in the double-scattering picture. It contains both central $\left(a \mathrm{e}^{b t}\right)$ and pheripheral (Bessel function) components, both of which are exactly crossing-symmetric. The peripheral part has a logarithmic energy-dependence, and since

$$
\begin{aligned}
& \left.J_{0}\left(R_{0} \sqrt{-t\left[\ln \nu-\frac{1}{2} i \pi\right.}\right]\right) \simeq J_{0}\left(R_{0} \sqrt{-t \ln \nu}\right)+i K \sqrt{-t} J_{1}\left(R_{0} \sqrt{-t \ln \nu}\right), \\
& K=\frac{\pi R_{0}}{4 \sqrt{\ln \nu}},
\end{aligned}
$$

$T_{\text {eff }}$ has a real part like a $J_{1}$ Bessel function; it therefore has a different phase from the imaginary $T_{\mathrm{el}}$ of eq. (2). This alters the phase of the absorptive correction, to give weak absorption of the real part of the $\rho$ pole amplitude.

\subsection{The "i-factor" model**}

In this model, absorptive cuts are calculated as follows: write Regge pole amplitudes with wrong-signature zeroes, and divide the signature factor $1+\tau \mathrm{e}^{i \pi \alpha}$ into two parts, 1 and $\tau \mathrm{e}^{-i \pi \alpha}$. Absorb these as in a conventional weak absorption model, then multiply the absorptive correction to the 1 part by $i$.

Appendix 2 describes how one can do this in an exactly crossing-symmetric way. This involves finding a Hermitian analytic, even function of $\nu\left(=\frac{1}{2}(s-u)\right)$ which is approximately equal to $\sqrt{i}$ in the energy range of interest (around $p_{\text {lab }} \simeq 6 \mathrm{GeV} / c$ ); the absorptive correction is then multiplied by this function. The extra energy dependence brought in by the $\sqrt{i}$ factor should be at most logarithmic (so as not to shift the branch point of the $j$-plane cut). It should not introduce any other unrealistic $j$-plane structure (such as fixed poles or dipoles), and, in accord with the duality postulate that low-lying singularities be small in FESRs, the function is required not

\footnotetext{
* See ref. [6].

** See ref. [7].
} 
to introduce any daughter absorptive cuts. This still leaves some freedom in the choice of the function, which obviously affects the final FESR result. I have tried several such crossing-symmetric $\sqrt{i}$ factors; the FESR results shown in fig. 4 are typical of the best agreement which I can obtain.

\subsection{FESR comparisons}

These three models have been used to evaluate numerically* the right-hand side of eq. (1) (the semi-circle integral) for s-channel helicity non-flip and flip amplitudes (denoted by $N$ and $F$ ) in $\pi^{-} \mathrm{p} \rightarrow \pi^{0} \mathrm{n}$. The left-hand side of the equation has been evaluated from the 1971 CERN phase shifts [15] with a cutoff** at $E_{\text {lab }}=2.1 \mathrm{GeV}$. The comparisons of left- and right-hand sides of eq. (1) (solid and dashed curves respectively) are shown in figs. $2-4$.

The basic strong absorption model (fig. 2) gives a qualitatively correct description of the non-flip FESR; it has a zero at approximately the correct $t$-value, caused by destructive pole-cut interference, which corresponds to the crossover zero in the high-energy amplitude. However, quantitatively the model does not have FESR duality. It gives an FESR too small by a factor of two in the forward direction, where the errors in the low-energy integral are very small (being determined only by the $\left[\pi^{-} p-\pi^{+} p\right]$ total cross section difference). The slope of the FESR in the forward direction is also poorly fitted.

The two new absorption models (Hartley-Kane and $i$-factor models) do not even qualitatively obey FESR duality (figs. 3 and 4); they give an FESR with the wrong sign in the forward direction, and with a flat, featureless $t$-dependence. They do not have the large slope at $t=0$, or the zero at $t \simeq-0.1$, which occur in the phase-shift integral.

I have made the same FESR comparison for two other typical new absorption models; these are a typical Regge-Regge cut model [8](containing a $\rho \otimes \mathbf{P}^{\prime}$ cut, but not*** an $\mathrm{A}_{2} \otimes \omega$ cut) and the model of Girardi [9] et al., which contains $\rho \otimes \mathrm{P} \otimes \mathrm{P}^{\prime}$ and $\omega \otimes \mathrm{P} \otimes \mathrm{A}_{2}$ cuts. In these two cases, the FESR results are essentially the same as for the Hartley-Kane and $i$-factor models. This suggests that the lack of FESR duality does not arise from small details of the models, but arises from general features which they have in common.

It seems likely that the same FESR disagreement will occur in other non-evasive non-flip vector-meson-exchange amplitudes; for instance, odd-signature $\mathrm{Kp}$ and $\pi \pi$ non-flip FESRs [18] show the same qualitative features as the $\pi^{-} p \rightarrow \pi^{0}$ non-flip FESR.

* The semi-circle in tegral is evaluated using the full (leading and non-leading) $j$-plane structure of the models. Later we shall check whether the non-leading contributions to the semi-circle integral are in fact small, as required by duality.

** I have also compared all the models with FESRs evaluated from the 1972 Saclay phase shifts [16], both with the CERN cutoff energy and with a higher cutoff at $E_{\text {lab }}=2.8 \mathrm{GeV}$. In all cases the results are qualitatively similar.

*** There are reasons to expect $[9,17]$ that $\rho^{\prime} \otimes P^{\prime}$ and $A_{2} \otimes \omega$ cuts cancel one another in $\pi^{-} \mathrm{p} \rightarrow \pi^{0} \mathrm{n}$. 
I have also compared the models with some continuous-moment sum rules [19]. CMSRs which are integrals of $\operatorname{Im} N$ show qualitatively the same effects as the FESRs, while CMSRs which are integrals of $\operatorname{Re} N$ show better agreement with the models.

(This can be simply explained in terms of $j$-plane structure, discussed in the next section.)

\section{Why are absorption models not dual?}

In this section we try to find a simple explanation of the dramatically undual behaviour of the absorption models in the non-flip $\pi^{-} \mathrm{p} \rightarrow \pi^{0}$ n FESR.

\section{1. j-plane structure}

Define the absorptive cut discontinuity $f(j, t)$ by

$$
\begin{gathered}
T(\nu, t)=T_{\text {pole }}(\nu, t)+\int_{-\infty}^{\alpha} \mathrm{c} d\left(\frac{\nu}{\nu_{1}}\right)\left[\frac{\nu_{0}^{2}-\nu^{2}}{\nu_{1}^{2}}\right]^{\frac{1}{2}(j-1)} f(j, t) \\
\equiv T_{\text {pole }}(\nu, t)+\int_{-\infty}^{\alpha_{\mathrm{c}}} \mathrm{d} j\left[G_{\mathrm{R}}(j, \nu)+i G_{\mathrm{I}}(j, \nu)\right] f(j, t)
\end{gathered}
$$

The weighting functions $G_{\mathrm{R}}(j)$ and $G_{\mathrm{I}}(j)$ are shown in fig. 5 , for $P_{\mathrm{lab}}=6.0 \mathrm{GeV} / c$, $\nu_{1}=m^{2}=0.88 \mathrm{GeV}^{2}$.

At fixed $t$, one can deduce $f(j)$ from $T(\nu)$ by a numerical method which is described in appendix 3 . I have used this method to examine the $j$-plane structure $f(j)$ of the various absorption models.

Fig. 6 shows the cut discontinuity of the basic SCRAM model in the non-flip amplitude at $t=0$ and $t=-0.5$. The pole contribution is shown as a $\delta$-function in $f(j)$. At $t=0$, the cut discontinuity is very sharply peaked near $j=0.5$, so it contributes roughly equally to real and imaginary parts of the amplitude $\left(G_{\mathrm{R}}(0.5)\right.$ and $G_{\mathrm{I}}(0.5)$ are equal, see fig. 5). This is the basic problem of the model - strong absorption in the imaginary part implies strong absorption in the real part.

Fig. 7 shows the $j$-plane structure of the Hartley-Kane model* at $t=0$. It differs from the basic SCRAM model by a large extra contribution in the region $0>j>-1$.

In fig. 8 are shown the cut discontinuities of the $i$-factor model (with the same $\sqrt{i}$ factor as was used for the FESR comparison, see appendix 2) at $t=0$ and at $t=-0.5$. Again, the dominant $j$-plane contributions are in the region $0>j>-1$.

* For non-zero momentum transfers the model has an essential singularity at the cut branch point, so one cannot extract the $j$-plane structure by the method of appendix 3 . However, since the absorptive amplitudes are smooth functions of $t, j$-plane structure at $t=0$ is relevant to a discussion of energy dependence at small $t$. 


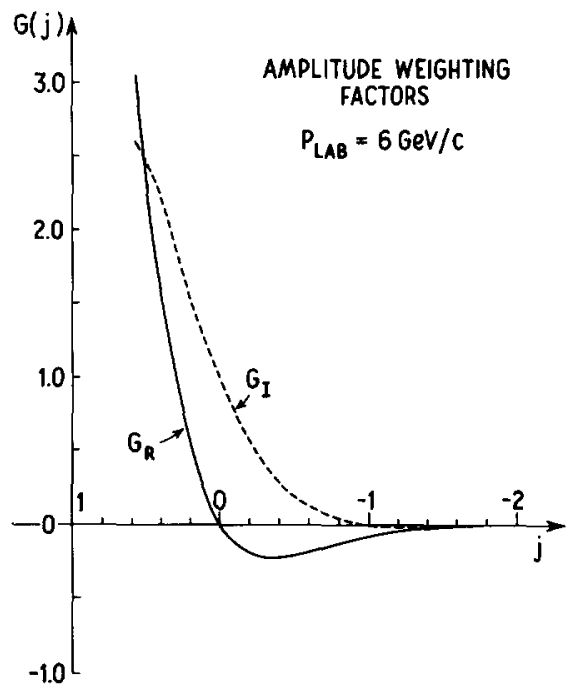

Fig. 5. The $j$-plane weighting function $G(j)$ as a function of $t$-channel angular momentum $j$. A cut amplitude at $p_{\text {lab }}=6 \mathrm{GeV} / c$ is an integral over $j$ of the cut discontinuity $f(j)$ weighted by $G(j)$ ( $G_{\mathrm{R}}$ for the real part of the amplitude, $G_{\mathrm{I}}$ for the imaginary part). $G$ is defined by eq. (5).

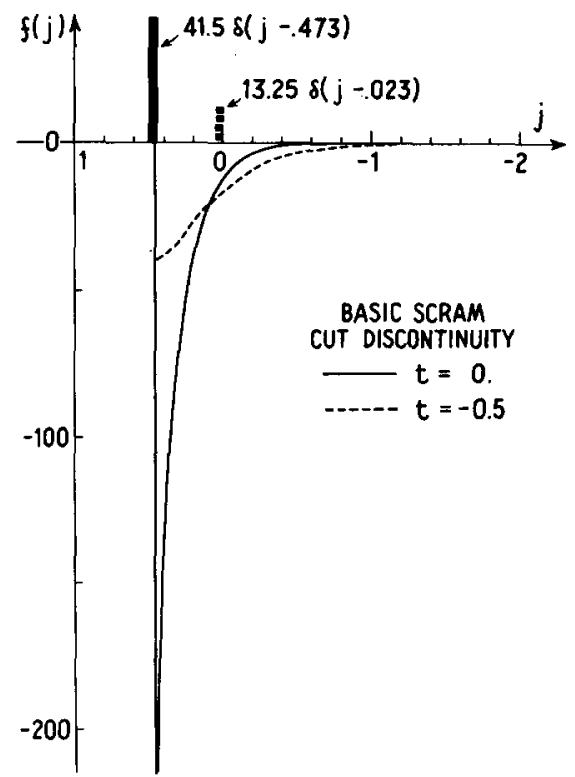

Fig. 6. The $j$-plane cut discontinuity $f(j)$ of the basic strong absorption model, at $t=0$ and $t=-0.5 . f(j)$ is defined by eq. (5). The $\rho$ pole is drawn as a $\delta$-function in the cut discontinuity. 


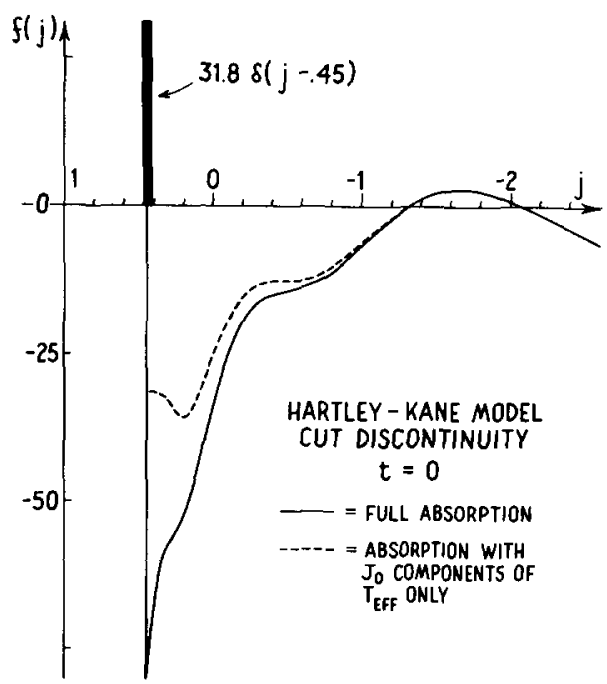

Fig. 7. As fig. 6, for the Hartley-Kane model at $t=0$. The solid curve is the full cut discontinuity, and the dashed curve is the discontinuity of the cut obtained by absorbing only with the "peripheral" Bessel function part of the effective elastic amplitude, eq. (3). The remainder (solid-dashed) is similar to the basic cut model, fig. 6 .
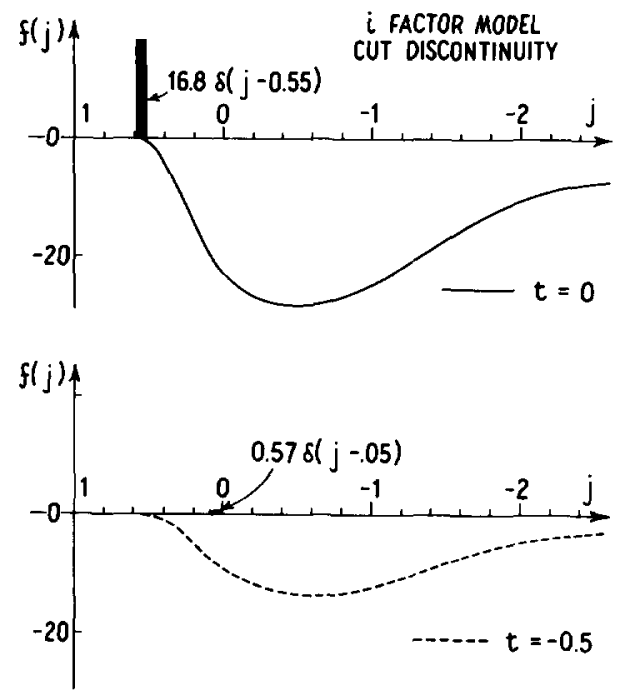

Fig. 8. As in fig. 6, for the Ringland et al. $i$-factor model, calculated as in appendix 2 , at $t=0$ and $t=-0.5$. 
The two other models which I have considered (a Regge-Regge cut model and the Girardi et al. RPR model) both involve extra $j$-plane cuts with branch points $\alpha_{c}(t) \simeq 0$. Therefore all four new absorption models contain a large extra $j$-plane contribution centred in the region between $j=0$ and $j=-1$.

It is easy to see why this is so, in terms of the weighting functions $G_{\mathbf{R}}(j)$ and $G_{\mathrm{I}}(j)$ in fig. 5. The models are designed to give strong absorption of imaginary parts, with weak absorption of real parts. The simplest way to do this is to take a basic absorption correction, and add an extra contribution which has opposite signs in real and imaginary parts of the amplitude. Thus the extra contribution must be in a $j$-plane region where $G_{\mathrm{R}}(j)$ and $G_{\mathrm{I}}(j)$ have opposite signs (i.e. where the Regge phase factor has $\operatorname{Re} / \operatorname{Im}<0$ ). The only such region is $0>j>-1$; so the extra contribution inevitably comes in this region.

The new absorption models therefore use a very wide region of $j$-plane singularities (between $j=0.5$ and $j=-1.0$ ) to explain the known $6 \mathrm{GeV} / c \pi^{-} \mathrm{p} \rightarrow \pi^{0} \mathrm{n}$ amplitude structure. This in itself suggests that the models will not be dual; as the energy changes, one expects contributions from different ends of this broad $j$-plane region to rapidly get out of step, producing a complicated energy dependence. We shall see later how this happens in detail.

For each small region of the $j$-plane cut, the FESR contribution (the semi-circle integral) can be calculated analytically, just as for a Regge-pole contribution. The FESR can therefore be written as an integral over the cut discontinuity:

$$
\begin{aligned}
& \text { FESR }=\text { pole }+\frac{\nu_{1}}{4 m} \int_{-\infty}^{\alpha_{c}} \mathrm{~d} j \frac{\sin \frac{1}{2} \pi(j+1)}{j+1}\left(\frac{N}{\nu_{1}}\right)^{j+1} f(j) \\
& \equiv \text { Pole term }+\int_{-\infty}^{\alpha_{\mathrm{c}}} \mathrm{d} j G_{\mathrm{s}}(j) f(j) .
\end{aligned}
$$

The FESR weighting function $G_{\mathbf{s}}(j)$ is shown in fig. 9. Using this figure, and figs. $6-8$, one can do the cut integral (eq. (6)) by hand to see which $j$-plane regions are most important in the FESR. From this it is obvious why the new models give the wrong sign in the non-flip FESR at $t=0$. The new component in $0>j>-1$ is heavily weighted in the FESR, and gives over $40 \%$ of the cut contribution. This enhanced cut over-cancels the pole, giving the wrong FESR sign. Note that the extremely lowlying part of the cut $(j<-1)$ is not important in the FESR; thus the same new component (in $0>j>-1$ ) causes both the agreement with $6 \mathrm{GeV} / c$ amplitudes and the disagreement with the FESR.

\subsection{Energy dependence}

The influence of the low-lying $j$-plane structure in the new models can be clearly seen by looking at the energy dependence of the amplitudes at intermediate energies. 


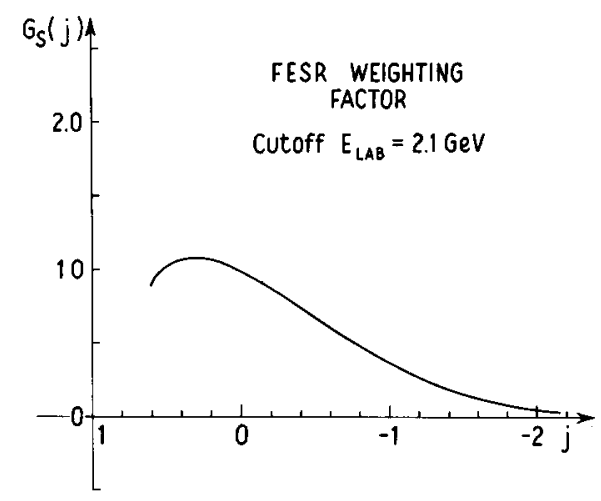

Fig. 9. The FESR weighting factor $G_{\mathrm{s}}(j)$, defined in eq. (6), as a function of angular momentum $j$. A $j$-plane cut contribution to the FESR is an integral of the cut discontinuity $f(j)$ weighted by $G_{\mathrm{s}}(j)$.

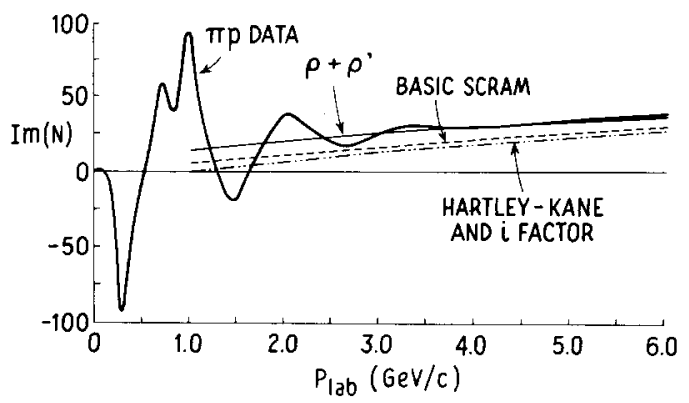

Fig. 10. The imaginary part of the forward non-flip amplitude $\operatorname{Im} N$ as deduced from total cross sections $\left[\sigma \mathrm{T}\left(\pi^{-} \mathrm{p}\right)-\sigma \mathrm{T}\left(\pi^{+} \mathrm{p}\right)\right]$ (thick solid curve) and in the models in this paper, as a function of pion lab momentum. Also shown is the Barger-Phillips $\rho+\rho^{\prime}$ pole model, which, unlike the absorption models, gives a good local average of the actual amplitude at low energies.

Figs. 10, 11 and 12 show respectively the energy dependence of the imaginary part of the non-flip amplitude $\operatorname{Im} N$ at $t=0$, the derivative of this quantity $\partial \operatorname{Im} N / \partial t$ at $t=0$, and the position in $t$ of the first zero of $\operatorname{Im} N$, in the various models. The figures also show the "experimental" values of these quantities, from total cross section data or from phase shifts at low energies.

In the Hartley-Kane and " $i$-factor" models, the low-lying $(0>j>-1)$ region of the cut grows larger (relative to the high-lying $\rho$ pole) as the energy decreases; thus at low energies the destructive cut overpowers the pole. With decreasing energy the zero of $\operatorname{Im} N$ moves in towards $t=0$ (fig. 12), and the value of $\operatorname{Im} N$ at $t=0$ becomes smaller relative to the experimental value from $\left(\sigma_{\mathrm{T}}\left(\pi^{-} \mathrm{p}\right)-\sigma_{\mathrm{T}}\left(\pi^{+} \mathrm{p}\right)\right)$ (fig. 10). 


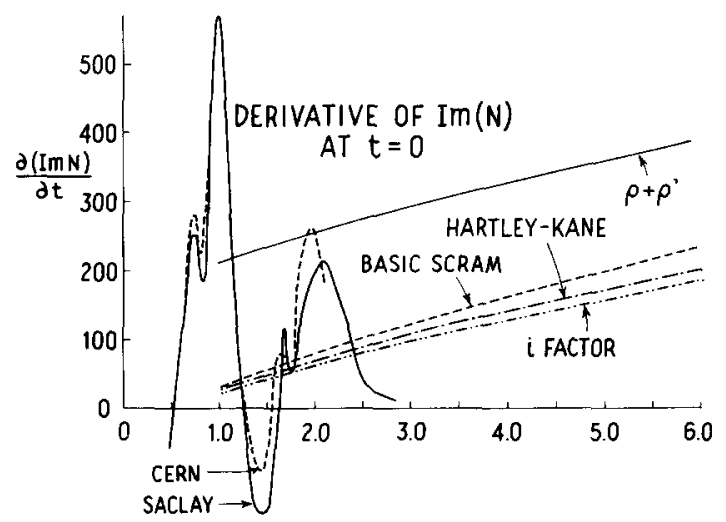

Fig. 11. The forward derivative of the imaginary part of the non-flip amplitude $\partial(\operatorname{Im} N) /\left.\partial t\right|_{t=0}$, as deduced from the Saclay 1972 phase-shift solution (thick solid curve), the CERN 1971 phase shift solution (thick dashed curve) and from the models in this paper. All models except the Barger-Phillips pole model give the local average of this quantity too low.

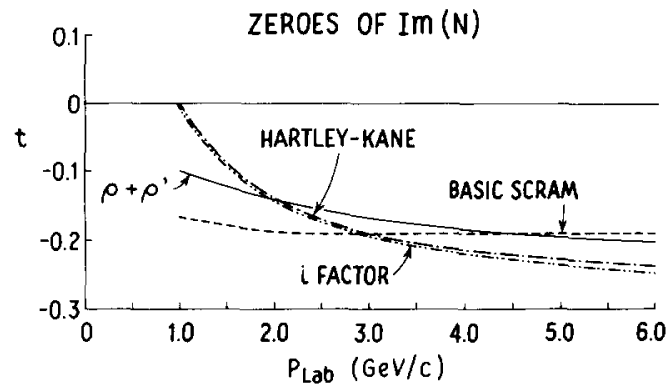

Fig. 12. The position in $t$ of the first zero of $\operatorname{Im} N$, as a function of lab momentum, in the highenergy models in this paper. The zeroes of $\operatorname{Im} N$ in the phase-shift solutions fluctuate too wildly for comparisons to be useful without some local averaging.

Therefore the models do not have local-average duality in $\operatorname{Im} N$; this is obviously connected to their lack of FESR duality*.

However, the basic strong cut model, with a fairly narrow $j$-plane structure (in $0.5 \sim j \sim 0$ ) shows a more constant energy dependence. At $t=-0.2$ the pole and cut have approximately the same energy dependence, so the zero of $\operatorname{Im} N$ does not move with energy. This naturally gives better FESR agreement.

Fig. 11 shows that the models are not dual in the local average of the quantity $\partial(\operatorname{Im} N) /\left.\partial t\right|_{t=0}$. (This is clearly connected to their failure to fit $\partial(\mathrm{FESR}) /\left.\partial t\right|_{t=0}$.) The derivative of the forward amplitude is mainly controlled by the high partial

* Although the new models give the correct $\operatorname{sign}$ of $\operatorname{Im} N$ at $t=0$ for energies above the FESR cutoff, they give the wrong sign in the FESR. This is possible because $\int \stackrel{\infty}{b} \operatorname{Im} N \mathrm{~d} \nu$ diverges. 
waves, and is therefore little affected by absorption [20] (a correction to low partial waves); this suggests that the Regge pole input should perhaps not be as simple as these models assume.

\subsection{Summary}

The non-duality of current Regge absorption models arises through a simple mechanism:

(i) In order to have strong absorption of the imaginary part of a vector-exchange amplitude, with weak absorption of the real part, new absorption models include a component which contributes with opposite signs to real and imaginary parts of amplitudes. To do this, the new component must be in the $j$-plane region $0>j>-1$.

(ii) The strength of absorption is designed to give correct amplitude structure at $p_{\text {lab }} \simeq 6 \mathrm{GeV} / c$. As the energy decreases, the low-lying cut component grows, relative to the high-lying $\rho$ pole, so that eventually the absorption over-cancels the $\rho$ pole in $\operatorname{Im} N$ at all $t$-values. This disagrees with local-average duality, and causes acute FESR disagreement at $t=0$.

There is one hidden assumption in this argument, which concerns $t$-dependence. The low-lying component is necessary in the region $t \simeq-0.3$, where $\pi^{-} \mathrm{p} \rightarrow \pi^{0} \mathrm{n}$ polarization data do not show the negative dip predicted by old absorption models. However, the main FESR disagreement occurs at $t=0$. So a model with a rapid variation in $t$ might get out of the difficulty. It is only because absorptive corrections come mainly in the low partial waves, and thus have a smooth $t$-dependence, that they give problems in the FESR at $t=0$.

Finally one may ask what has become of the qualitative duality (that Regge and resonance amplitudes are both concentrated in the peripheral partial waves, and there fore have similar $t$-dependences) which absorption models are believed to possess. Even at high energies, the new absorption models do not have a simple partial wave structure - for instance, the central partial waves of the pole amplitudes are not simply absorbed away, they are multiplied by a complex factor (because of the extra phase of the absorbing amplitude). As the energy decreases, the absorbing amplitude $T_{\text {eff }}$ becomes larger, tending to produce over-absorption of central partial waves; furthermore, the Regge pole contribution to peripheral partial waves becomes smaller, because of shrinkage. Therefore at low energies one does not expect the new absorption models to show the simple peripheral impact parameter structure. This is why they are not dual to the peripheral low energy resonances.

\section{Possible dual absorption models}

We have shown that current absorption models are not dual, and have given a simple reason why this is so, in terms of their $j$-plane structure. The next step is not to abandon the models, but to try to incorporate their best features in a new model 
which can describe both the FESRs and the high energy amplitudes. One can try altering the models in any of three general directions:

(a) Alter the Regge pole input.

(b) Alter the absorption.

(c) Do not alter the models to leading order in $\nu$, but add extra low-lying singularities to fit the FESRs.

\subsection{Altered Regge pole input}

This choice can probably be made to work, but would certainly involve extra complications - since present models use the simplest possible $\rho$ pole input. In particular, the behaviour of $\partial(\mathrm{FESR}) /\left.\partial t\right|_{t=0}$ and $\partial(\operatorname{Im} N) /\left.\partial t\right|_{t=0}$ may indicate curvature of the $\rho$ trajectory [20,21]. A $\rho^{\prime}$ pole may contribute [22]; however, if it is too low-lying (say $\alpha(0) \simeq-\frac{1}{2}$ ), it might give the same FESR problems as the low-lying $(0>j>-1)$ component of the new absorption models.

\subsection{Altered absorptive corrections}

The absorption models which do not fit the $\pi^{-} \mathrm{p} \rightarrow \pi^{0}$ n FESR use an absorptive correction which is a smooth function of $t$, which is broad in the $j$-plane, and which is unshrinking. (In the Barger-Phillips $\rho+\rho^{\prime}$ Regge pole model [19], the amplitude has none of these properties, but agrees well with FESR and local-average duality, as is shown in figs. 10-12.) One can therefore try to construct new cut models, discarding some of the above three properties, in order to correctly describe the FESR. For instance, Johnson $[14,23]$ has proposed a model (which fits the CMSRs) in which the "absorptive" correction has a sharp $t$-dependence and vanishes in the forward direction. This form of correction changes the high partial waves of the Regge pole input, and is therefore a radical modification of the original absorption idea.

Another possibility is suggested by the observation that the non-flip $\pi^{-} p \rightarrow \pi^{0} n$ FESR (fig. 2) has a $t$-dependence very similar to the imaginary part of the high energy amplitude [4]. The Regge absorption models do not reproduce this simple feature, because pole and cut contributions have different energy dependences, and get out of step as the energy changes. This suggests that the absorption should be forced to have the same energy as the $\rho$ pole, at all momentum transfers. Such a picture is confirmed by $K^{ \pm}$p elastic cross-sections, which show that the imaginary part of the non-flip $\omega$-exchange amplitude has Regge-pole-like shrinkage $[3,24]$. Since this amplitude contains strong absorptive corrections, it again suggests that the absorptive amplitude should have a pole-like energy dependence. Furthermore, the most recent measurements [25] show that the $\pi^{-} \mathrm{p} \rightarrow \pi^{0} \mathrm{n}$ polarization is small over a wide range of momentum transfers - implying that Regge pole and absorption amplitudes have similar phases (as well as similar energy dependence) over a wide $t$-range. The results of Elvekjaer, Inami and Ringland [28] (who have examined phase shift amplitudes at $p_{\text {lab }} \sim 2.5 \mathrm{GeV} / c$ ) suggest that this picture may even be valid out to $t \simeq-2.5$. 
One is therefore encouraged to construct a "shrinking cut" model in which both the phase and the energy dependence of the absorptive correction are closely matched to that of the Regge pole. The $t$-dependence of $\operatorname{Im} N$ at $6 \mathrm{GeV} / c$ is still produced by destructive pole-cut interference, with the absorptive cut mainly confined to the central partial waves. It seems very likely that this model would easily fit both the high-energy amplitudes and the sum rules in $\pi^{-} \mathrm{p} \rightarrow \pi^{0} \mathrm{n}$. There are obviously many $j$-plane structures which would give the required phase and energy dependence over some finite energy range; however, the simplest choice is to make the cut discontinuity peak sharply near the $\rho$ pole position. This form of cut has been discussed in the context of complex pole models [26]; in fact arguments based on $t$-channel unitarity often tend to give a cut discontinuity which peaks near a pole.

In such a model, all the important singularities are concentrated in a small $j$-plane region near the $\rho$ pole. This guarantees a simple energy dependence over a wide energy range, in agreement with duality.

The dual absorption model [29] offers another possibility, in which imaginary parts of amplitudes are forced to have a peripheral $t$-dependence, and the full amplitude usually satisfies FESRs by construction. However, it may contain right-signature fixed poles or other complicated $j$-plane structure; the role of these in an eventual complete model is not clear.

\subsection{Models with low-lying j-plane structure}

It is obviously possible to fit the FESRs by starting from an undual Regge model, leaving it unchanged to leading order in $\nu$, and adding to it some low-lying singularities which are important in FESRs but unimportant in the high-energy amplitude. If such a modification to one of the new absorption models is to be successful, then it must involve extra $j$-plane singularities which are extremely low-lying (in these models, the whole region $j \geqslant-1$ is used to describe the high-energy amplitude; so the extra singularities must appear in $j<-1$ ). It is therefore a question of adding granddaughters, rather than daughters. These grand-daughters must also be extremely large, since they significantly change both the amplitude at intermediate energies, and the FESR.

Such a solution, if true, would have very serious consequences for duality. The local average of the amplitude at low-intermediate energies would no longer be given by the leading crossed-channel singularities, so that FESRs would no longer be a useful constraint on these singularities. It would imply that the apparently simple energy dependence of amplitudes (on which duality is based) is in fact caused by a complicated sum of high-lying and low-lying effects. Unless one could find some simple constraints on the low-lying singularities, the continuation from high to low energies would be arbitrary, and duality would lose all predictive power.

It might be possible to introduce these low-lying singularities, in a way that is not completely arbitrary, by using a different definition of the absorption prescription. There are several such definitions, all of which are equivalent in the high-energy 
limit, but which differ at low energies. For the FESR tests I used the form of absorption which gives the simplest possible $s-u$ crossing properties and $j$-plane structure; absorption was defined by convolutions in a transverse momentum whose relation to the momentum transfer, $t$, is independent of energy (and therefore gives exactly $s-u$ crossing-symmetric, daughterless absorptive cuts). This is equivalent to absorp* tion by multiplying amplitudes at fixed impact parameter, $b$ (see appendix 2). However, one could also define absorption by multiplication of partial-wave amplitudes; the two would be identical at high energies, but the second form might give a better continuation to low energies because of daughter effects. I have tried several different methods, including partial-wave absorption; but I have not yet found a way to improve the low-energy amplitude behaviour, without losing $s-u$ crossing symmetry or losing Regge behaviour for some complex s-values. The reasons for this are given in appendix 4.

\subsection{Future tests}

These schemes are all designed to describe the $\pi^{-} \mathrm{p} \rightarrow \pi^{0} \mathrm{n}$ FESRs and amplitudes at $6 \mathrm{GeV} / c$; they are therefore all forced to have rather similar amplitude structure in the $2-6 \mathrm{GeV}$ energy range. However, at higher energies the differences between the various models become clearer. The energy dependence of the imaginary part of the non-flip amplitude, deduced from $\mathrm{K}^{ \pm} \mathrm{p}$ or $\pi^{ \pm} \mathrm{p}$ elastic cross section differences, will be useful in deciding between models. $\pi^{-} \mathrm{p} \rightarrow \pi^{0} \mathrm{n}$ polarization data at higher energies will also be a useful test; models of type (c), with a broad $j$-plane structure, predict a polarization which develops a negative bump at high energies, as in the basic strong cut model. However, in the "shrinking cut" picture the polarization is more constant with energy - and so should remain positive or zero to quite high energies. Finally, $\pi^{-} \mathrm{p} \rightarrow \pi^{0} \mathrm{n}$ cross sections at high energies will be another useful constraint (mainly on the spin-flip amplitude).

I have only discussed these possible developments of the absorption model in the context of pion-nucleon charge-exchange; however, they will only be useful in the long run if they form part of a unified picture of all high-energy two-body amplitudes. To discuss this goes beyond the scope of this paper. However, two remarks are worth making:

(i) The property of duality (of simple energy dependence down to unexpectedly low energies) has been approximately confirmed in amplitudes for many processes. Therefore it seems worthwhile to try to explain a simple energy dependence in as simple a way as possible; in particular, not to use broad $j$-plane structures, which tend to have a complicated energy dependence.

(ii) High energy amplitudes (and cross sections) can be divided into two classes; those which show Regge pole-like shrinkage, and those which do not. $\pi^{-} \mathrm{p} \rightarrow \pi^{0} \mathrm{n}$ amplitudes belong to the (smaller) first class which do shrink. The results of this paper are not directly relevant to the larger class of processes, such as photoproduction and vector meson production, which do not show obvious shrinkage. For instance, 
the "shrinking cut" picture is probably not useful in pion photoproduction, where the old strong absorption model gives a good description [27] of both high energy data and FESRs. To produce a unified picture of both shrinking and unshrinking amplitudes requires a detailed study of the energy dependence and duality properties of a wide variety of amplitudes.

\section{Conclusions}

In this paper we have examined the duality properties of various Regge absorption models in pion-nucleon charge exchange, using FESRs to test the models. We have mainly been interested in the new forms of the absorption model; in which the absorptive phase is altered in order to agree with known amplitude analysis results. We found that all of these models give the same striking disagreement with FESR duality in the $s$-channel helicity non-flip amplitude. This is especially surprising in view of the well-known qualitative duality of absorptive zero structure in this amplitude.

In order to compare Regge models with the FESRs, we had to continue them down in energy from the high-energy region where they are primarly used. One may ask whether this continuation is unique - or whether some other form of continuation would give better agreement with low-energy amplitudes and FESRs. The method of continuation was chọsen to minimise the contribution of low-lying $j$-plane structure (daughters etc.), so that the amplitudes have the natural energy dependence of the leading $j$-plane singularities of the models. It might be possible to fit the FESRs by extrapolating down with large "daughter" singularities, which are unimportant at high energies, yet dominate the FESRs (i.e. they effectively decouple the high- and low-energy regions). If this solution were correct, it would have disturbing implications for duality - for instance, it implies that FESRs are not a useful constraint on the Regge singularities which dominate the high-energy amplitude.

It turns out that the lack of duality does not arise from details specific to the two models which are tested in this paper (the Hartley-Kane model and the $i$-factor model), but arises through a basic general mechanism which is common to a large class of models. This mechanism is most simply understood in terms of $j$-plane structure.

The basic strong absorption model has an absorptive cut discontinuity which peaks sharply near the branch point, $\alpha_{c}(t) \simeq 0.5$. Thus the absorption contributes with equal strength to real and imaginary parts of the amplitude, in disagreement with recent amplitude analysis results. The model has therefore been modified (in several different ways), to give strong absorption of imaginary parts and weak absorption of real parts. The new models do this by adding a large new component which contributes with opposite signs to real and imaginary parts. To have the correct Regge phase $\left(1-\mathrm{e}^{-i \pi \alpha}\right)$ to do this, the new component must come in the $j$-plane region $0>j>-1$ (although its theoretical motivation is very different in the various models). Since their $j$-plane singularities are so widely spread out, the new models tend to have a more complicated energy dependence, which conflicts with the simple (dual) energy 
dependence of the actual amplitudes. As the energy decreases, the low-lying component becomes relatively more important in the model amplitudes eventually overcancelling the $\rho$ pole contribution. This causes disagreement with both FESR and localaverage duality.

In their presently accepted forms, therefore, the ideas of absorption and duality are not compatible. There are many possible solutions to this difficulty. For instance one might assume that duality is only applicable to Regge pole amplitudes -- the "Born terms" of some perturbation expansion - and that higher-order effects, such as cuts, are not dual. Then in comparing cut amplitudes with FESRs, one would have to include large low-lying contributions. Since so many amplitudes show strong absorption-like effects, this would mean that duality is not directly applicable to most Regge amplitudes. Alternatively, one might abandon the idea of absorption altogether and try to construct Regge amplitudes from some completely different viewpoint. However, current absorption ideas are so useful in understanding many features of high-energy amplitudes, that it seems unwise to abandon them. It seems better to try to improve the models, in the light of the FESR results; this may produce an absorption model with better duality properties, and at the same time a more realistic high energy amplitude structure. Many such modifications to current models are possible. Their main drawback is that they all lack theoretical motivation, so are not compelling unless experimentally confirmed in many amplitudes. Even so, they may be a step towards a Regge absorption model which is realistic at high energies, is theoretically motivated, and is dual to low-energy amplitudes.

I should like to thank Gordy Kane for his hospitality at the University of Michigan, where most of this work was completed. I am grateful to many people for useful comments and discussions, particularly Frank Henyey, Gordy Kane, Marc Ross, Geoffrey Fox, Chris Quigg, D.P. Roy, Gordon Ringland and Roger Phillips.

\section{Appendix 1. Amplitudes and sum rules}

For the process $\pi^{-} \mathrm{p} \rightarrow \pi^{0} \mathrm{n}$, number the particles $\pi_{1}^{-} \mathrm{p}_{2} \rightarrow \pi_{3}^{0} \mathrm{n}_{4}$ and label their momenta $p_{i}(i=1, \ldots, 4)$ accordingly. Then the Mandelstam variables are

$$
\begin{array}{ll}
s=\left(p_{1}+p_{2}\right)^{2}, & t=\left(p_{3}-p_{1}\right)^{2}, \\
u=\left(p_{4}-p_{1}\right)^{2}, & s+t+u=2 m^{2}+2 \mu^{2} ;
\end{array}
$$

$m$ is the nucleon mass, and $\mu$ is the pion mass. Define

$$
\nu=\frac{1}{2}(s-u), \quad \omega=\frac{s-u}{4 m}=\frac{\nu}{2 m} .
$$

I shall use amplitudes $N$ and $F$, which are related to CGLN amplitudes [33] as follows: 


$$
N=2\left(A^{-}+\omega B^{-}\right), \quad F=2 \sqrt{\frac{-t}{4 m^{2}}} A^{-}
$$

$N$ and $F$ have no kinematic singularities in $\nu$ at fixed $t$, and are odd functions of $\nu$ $(N(v, t)=-N(-v, t)$, etc. $)$. In the large $s$, finite $t$ limit, $N$ and $F$ are equal to $s$-channel helicity non-flip and flip amplitudes respectively, so the expressions for experimental quantities become

$$
\begin{aligned}
& \sigma_{\mathrm{T}}\left(\pi^{-} \mathrm{p}\right)-\sigma_{\mathrm{T}}\left(\pi^{+} \mathrm{p}\right)=\left.\frac{0.389}{p_{\mathrm{lab}}} \operatorname{Im} N\right|_{t=0} \quad \mathrm{mb}, \\
& \left(\frac{\mathrm{d} \sigma}{\mathrm{d} t}\right)_{\pi^{-} \mathrm{p} \rightarrow \pi^{0} \mathrm{n}}=\frac{0.389 m^{2}}{32 \pi k^{2}}\left(|N|^{2}+|F|^{2}\right) \quad \mathrm{mb} / \mathrm{GeV}^{2}, \\
& P_{\pi^{-} \mathrm{p} \rightarrow \pi^{0_{\mathrm{n}}}}=\frac{2 \operatorname{Im}\left(N F^{*}\right)}{|N|^{2}+|F|^{2}},
\end{aligned}
$$

where $p_{\text {lab }}$ is the lab momentum of the initial pion, and $k$ is the c.m. momentum.

Denoting $N$ or $F$ by $T$, the continuous moment sum rules are defined by

$$
S(n, t)=\frac{1}{2} \int_{\omega_{0}}^{N} \operatorname{Im}\left[\left(\omega_{0}^{2}-\omega^{2}\right)^{\frac{1}{2} n} T(\omega, t)\right] \mathrm{d} \omega+\text { Born term }
$$

The FESRs are the special case when $n=0$. Thus the non-flip FESR at $t=0$ is given by

$$
S(0,0)=\frac{1}{2 \times 0.389} \int_{\omega_{0}}^{N} p_{1 \mathrm{ab}}\left[\sigma_{\mathrm{T}}\left(\pi^{-} \mathrm{p}\right)-\sigma_{\mathbf{T}}\left(\pi^{+} \mathrm{p}\right)\right] \mathrm{d} E_{\mathrm{lab}}+\text { Born term }
$$

The FESRs were evaluated numerically from the CERN 1971 and Saclay 1972 phase shifts. Curves shown are for the CERN phase-shifts with a cutoff at $E_{\text {lab }}=2.10 \mathrm{GeV} / c$. These phase-shifts use a truncated partial wave series. However, a quantity of particular interest is the derivative of the non-flip FESR at $t=0-$ since the Regge models seem to give a poor description of this quantity. The derivative at $t=0$ is strongly affected by the high partial waves of the phase-shift amplitudes - so the truncation of the;partial wave series may affect the value of the derivative. It is hoped in the near future to evaluate the FESRs using Alcock and Cottingham's estimates [30] for the high partial waves to give more definite information on the duality of $\partial(\operatorname{Im} N) / \partial t$.

The choice of zero-moment FESRs out of the infinity of possible sum rules was arbitrary - one can also look at other moments of CMSR (this has been done, see sect. 3) or try to construct an "optimised" FESR [31]. However, optimised FESRs have only been constructed for cases where the high-energy amplitude is approximately by an effective Regge pole, and have been used to estimate the residue of this 
pole (thus to predict high energy amplitudes). Our purpose is quite different - we want to test high-energy models in which the $j$-plane structure is known to be widely spread out (between $j=0.5$ and $j=-1.0$ ). Therefore we cannot use effective pole approximations. In this case it is important that the sum rule samples different regions of the $j$-plane structure of the model evenly - i.e. that the sum rule $j$-plane weighting function $G_{\mathrm{s}}(j)$ (see sect. 4) be a smooth function of $j$, which does not unfairly deemphasize certain $j$-plane regions of the Regge model. The FESR weighting function $G_{\mathrm{s}}(j)$ (fig. 9) is indeed smooth and without sign changes - so that a zero moment FESR samples $j$-plane structure evenly, and thus is probably close to being an "optimised" FESR for our purpose.

\section{Appendix 2}

For all models, the $\rho$ pole amplitude is written in the form

$$
\begin{aligned}
& N=A_{\mathrm{N}}(t)\left(\frac{\nu}{\nu_{\mathrm{N}}}\right)\left[\frac{\nu_{0}^{2}-\nu^{2}}{\nu_{\mathrm{N}}^{2}}\right]^{\frac{1}{2}(\alpha-1)}, \\
& F=\sqrt{-t} A_{\mathrm{F}}(t)\left(\frac{\nu}{\nu_{\mathrm{F}}}\right)\left[\frac{\nu_{0}^{2}-\nu^{2}}{\nu_{\mathrm{F}}^{2}}\right]^{\frac{1}{2}(\alpha-1)},
\end{aligned}
$$

when $\alpha$ is the trajectory function and $\nu_{0}$ is taken to be $2 m \mu$ at all momentum transfers. $N$ and $F$ are denoted in common by $T$.

Cut amplitudes are calculated by convolutions in a space of two-dimensional transverse momenta $q$, related to momentum transfers by $t=-|q|^{2}$. The amplitudes are defined to have their standard Regge phase on the line $q_{\mathrm{y}}=0$. For other $q$ values the phase is given by angular-momentum conservation:

$$
T(\nu, q)=T\left(\nu, t=-|q|^{2}\right) \mathrm{e}^{i n \phi} ;
$$

$\phi$ is defined in fig. 13 and $n$ is the net $s$-channel helicity flip, $n=\lambda_{1}+\lambda_{4}-\lambda_{2}-\lambda_{3}$. In terms of fig. 13 the convolution of two amplitudes $T_{1}$ and $T_{2}$ is defined by:

$$
H(\nu, q)=T_{1} \otimes T_{2}=\int \mathrm{d}^{2} q_{1} T_{1}\left(\nu, q_{1}\right) T_{2}\left(\nu, q_{2}\right)
$$

If amplitudes are defined as functions of impact parameter, $b$, by the Fourier-Bessel transform

$$
T_{i}(\nu, b)=\int_{0}^{\infty}|q| \mathrm{d}|q| J_{n}(b|q|) T_{i}\left(\nu, t=|q|^{2}\right),
$$




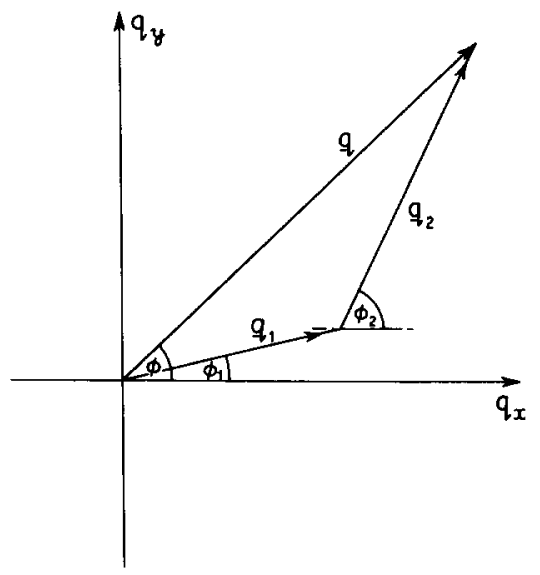

Fig. 13. Two-dimensional transverse momenta $q$ in the amplitude convolution used to define absorption. $|q|^{2}=-t$.

then by the convolution theorem, the above definition of absorption is equivalent to multiplication of amplitudes at constant impact parameter:

$$
H(\nu, b)=2 \pi T_{1}(\nu, b) T_{2}(\nu, b) .
$$

Note that the convolution is done at constant $\nu$, rather than at constant energy. This means that if $T_{1}$ and $T_{2}$ have exact symmetry under $\nu \rightarrow-\nu$, so will their convolution. For simple forms of $T_{1}$ or $T_{2},($ A.2.3) can be simplified to a single integral, or done analy tically.

\section{A.2.1. The basic SCRAM model}

(This is based on the model described in ref. [11]. Parameters are chosen to give the best fit to $6 \mathrm{GeV} / c$ amplitudes at small-medium $t$ values.) The $\rho$ pole amplitude is defined by

$$
\begin{aligned}
& A_{N}=\frac{m_{\rho}^{2}}{m \sqrt{2}} \frac{1}{t-m_{\rho}^{2}} \gamma_{++}, \quad A_{F}=\frac{m_{\rho}^{2}}{m \sqrt{2}} \frac{1}{t-m_{\rho}^{2}} \gamma_{+-}, \quad \text { (A.2.6) } \\
& \alpha=0.47+0.9 t, \quad \nu_{\mathrm{N}}=\nu_{\mathrm{F}}=0.31, \quad \gamma_{++}=-22.6, \quad \gamma_{+-}=85.7 .
\end{aligned}
$$

The absorptive cut is given by 


$$
T_{\text {cut }}(\nu, t)=\frac{m}{4 \pi^{2}}\left(\nu_{0}^{2}-\nu^{2}\right)^{-\frac{1}{2}} T_{\mathrm{el}} \otimes T_{\text {pole }},
$$

where the elastic amplitude consists of a purely non-flip pomeron term:

$$
T_{\text {el }}=-\left(\nu_{0}^{2}-\nu^{2}\right)^{\frac{1}{2}} \frac{g}{2 m} \mathrm{e}^{\frac{1}{2} a t},
$$

$g$ corresponds roughly to the pion-nucleon total cross section in $\mathrm{GeV}$ units. We have taken

$$
g=\frac{27}{0.389}, \quad a=7.0 .
$$

The cuts are then multiplied by real, energy independent $\lambda$ factors, $\lambda_{N}$ and $\lambda_{F}$ for non-flip and flip amplitudes respectively:

$$
\lambda_{N}=1.84, \quad \lambda_{F}=1.65 .
$$

\section{A.2.2. The Hartley-Kane model*}

The $\rho$ pole amplitude is given by

$$
\begin{aligned}
& A_{N}=\frac{-2}{m} \beta_{++} \Gamma\left(\frac{1-\alpha}{2}\right), \quad A_{F}=\frac{2}{m} \beta_{+-} \Gamma\left(\frac{1-\alpha}{2}\right), \\
& \alpha=0.45+t, \quad \nu_{N}=\nu_{F}=0.46, \quad \beta_{++}=-3.50, \quad \beta_{+-}=-13.50 .
\end{aligned}
$$

The absorption is as in eq. (A.2.7), with $T_{\mathrm{el}}=T_{\mathrm{P}}+T_{\mathrm{D}}$, and

$$
\begin{aligned}
& \left.T_{\mathrm{P}}=\frac{-1}{2 m}\left(\nu_{0}^{2}-\nu^{2}\right)^{\frac{1}{2}}\left[\mathrm{ge}^{d t}+a_{0} \mathrm{e}^{b_{0} t} J_{0}\left(c_{0} \sqrt{-\frac{1}{2} t \ln \left(\nu_{0}^{2}-\nu^{2}\right.}\right)\right)\right], \\
& \left.T_{\mathrm{D}}=\frac{-1}{2 m}\left(\nu_{0}^{2}-\nu^{2}\right)^{\frac{1}{2}}\left[a_{1} \mathrm{e}^{b_{1} t} J_{0}\left(c_{1} \sqrt{-\frac{1}{2} t \ln \left(\nu_{0}^{2}-\nu^{2}\right.}\right)\right)\right], \\
& g=35.0, \quad a_{0}=29.4, \quad c_{0}=2.55, \quad d=1.7, \quad b_{0}=2.0, \\
& a_{1}=59.0, \quad c_{1}=2.04, \quad b_{1}=2.0 .
\end{aligned}
$$

The elastic amplitude is purely helicity-non-flip.

The cut integrals are truncated at $t=-4.0$ to avoid divergences from the gamma functions. Contributions from the region $t \simeq-4.0$ are very small, so the amplitude is insensitive to the cutoff $t$-value.

\footnotetext{
${ }^{*}$ See ref. [6].
} 


\section{A.2.3. The i-factor model ${ }^{*}$}

The $\rho$ pole contribution in this model is given by

$$
\begin{aligned}
& A_{N}=\frac{8 \pi}{m} \gamma_{+} \Gamma(1-\alpha)\left(2 \sin \frac{1}{2} \pi \alpha\right), \quad A_{F}=\frac{4 \pi}{m^{2}} \gamma_{-} \Gamma(1-\alpha)\left(2 \sin \frac{1}{2} \pi \alpha\right),(\mathrm{A} .2 .13) \\
& \alpha=0.55+t, \quad \nu_{N}=0.573, \quad \nu_{F}=0.975, \quad \gamma_{+}=-0.165, \quad \gamma_{-}=2.15
\end{aligned}
$$

Conventional absorptive cuts would be given by eqs. (A.2.7) and (A.2.8), again truncated at $t=-4.0$, with

$$
g=\frac{15.6}{0.389}, \quad a=5.8 \text {. }
$$

However, to calculate cuts in this model, first replace the factor $2 \sin \frac{1}{2} \pi \alpha$ in the $\rho$ pole amplitude by $2 \sin \frac{1}{2} \pi\left(\alpha+\frac{1}{2}\right)$. Calculate the absorptive cut (eqs. (A.2.7) and (A.2.14)) from this modified $\rho$ pole amplitude, then multiply the resulting cut by $\sqrt{i}$. This is completely equivalent to the original method [7] of splitting the signature factor into two parts, then multiplying one part by $i$. However, it is easier to make crossing-symmetric, by writing the $\sqrt{i}$ factor in a crossing-even Hermitian analytic form. The following forms have been tried: Setting

$$
\begin{array}{ll}
y=\left(\frac{\nu_{0}^{2}-\nu^{2}}{\nu_{1}^{2}}\right), & n=1,2,3,4 \ldots, \\
\sqrt{i} \simeq A^{n}\left(\frac{1}{2} \ln y\right)^{-n}, & \nu_{1}=11.0 \exp [-A \cos (\pi / 4 n)], \\
A=\frac{\pi}{2 \sin (\pi / 4 n)}, & \nu_{1}=3.8, \\
\sqrt{i} \simeq \pi \sqrt{2}\left[\frac{1}{\ln y}-\frac{1}{y-1}\right], & \nu_{1}=11.0, \\
\sqrt{i} \simeq \frac{\pi}{\sqrt{2} \ln y}\left[1-y^{-\frac{1}{2}}\right], & \nu_{1}=2.3, \\
\sqrt{i} \simeq \frac{\pi \sqrt{2}}{0.955 \ln y}\left[1-y^{-1}\right], & \nu_{1}=11.0 . \\
\sqrt{i} \simeq \frac{1}{\sqrt{2}}\left[1+y^{-\frac{1}{2}}\right], &
\end{array}
$$

Forms (A.2.16)-(A.2.18) are designed so as not to have an extra $1 / \log \nu$ pole in the small $\nu$ region. They do this by introducing daughter cuts. The FESR comparisons

\footnotetext{
* See ref. [7].
} 
and $j$-plane structures shown in the figures use form (A.2.15) with $n=2$. Other "daughterless" forms have not yet given significantly better FESR agreement. Absorptive corrections are not included in the spin-flip amplitude $F$.

\section{A.2.4. Crossing symmetry}

All the amplitude expressions in this appendix have exact anti-symmetry under $\nu \rightarrow-\nu$, and are Hermitian analytic (there is a region between the cut branch points $\nu= \pm \nu_{0}$ where they are real). They are therefore suitable for FESR applications.

\section{Appendix 3. $j$-plane analysis}

Suppose one is given a method of calculating a function $F(\nu, t)$ which is odd in $\nu$, and which is known to arise from a $j$-plane cut with branch-point $\alpha$ and a discontinuity which can be parametrised as a smooth function of $j$. Then define the cut discontinuity $f(j, t)$ by

$$
F(\nu, t)=\int_{-\infty}^{\alpha_{\mathrm{c}}} \mathrm{d} j\left(\frac{\nu}{\nu_{1}}\right)\left[\frac{\nu_{0}^{2}-\nu^{2}}{v_{1}^{2}}\right]^{\frac{1}{2}(j-1)} f(j, t) .
$$

(From now on we shall assume a fixed value of $t$ and suppress all references to $t$.) Then we wish to approximate $f(j)$ by a form $\widetilde{f}(j)$, which is a sum of known functions:

$$
f(j) \simeq \widetilde{f}(j)=\sum_{i=1}^{N} c_{i} g_{i}(j) .
$$

Then defining

$$
\begin{aligned}
& G_{i}(\nu)=\int_{-\infty}^{\alpha_{c}} \mathrm{~d} j\left(\frac{\nu}{\nu_{1}}\right)\left[\frac{v_{0}^{2}-\nu^{2}}{v_{1}^{2}}\right]^{\frac{1}{2}(j-1)} g_{i}(j), \\
& F(\nu) \simeq \widetilde{F}(\nu)=\sum_{i=1}^{N} c_{i} G_{i}(\nu) .
\end{aligned}
$$

We want to choose the coefficients $c_{i}$ so that the approximation $\widetilde{F}$ resembles the original form $F$ as closely as possible over a wide energy range. Then the expression (A.3.2) gives a good approximation to the cut discontinuity $f(j)$.

This is a simple linear minimization problem whose solution is well known to be given by a single matrix inversion. To fill in the details: 
Let $F=A+i B, \widetilde{F}=\widetilde{A}+i \widetilde{B}$, where $A, B, \widetilde{A}, \widetilde{B}$ are real functions. Then define a $\chi^{2}$

$$
\chi^{2}=\sum_{m=1}^{M} U_{m}\left[A\left(\nu_{m}\right)-\widetilde{A}\left(\nu_{m}\right)\right]^{2}+V_{m}\left[B\left(\nu_{m}\right)-\widetilde{B}\left(\nu_{m}\right)\right]^{2} ;
$$

$U_{m}, V_{m}$ are real positive weights associated with the (possibly complex) energies $\nu_{m}$. $\chi^{2}$ is to be minimized by varying the coefficients $c_{i}$. Setting all derivatives

$\partial \chi^{2} / \partial c_{i}=0$ gives the matrix equation

$$
\sum_{l} M_{k l} c_{l}=R_{l}
$$

where

$$
\begin{aligned}
R_{k} & =\sum_{m} U_{m} A\left(\nu_{m}\right) D_{k}\left(\nu_{m}\right)+V_{m} B\left(\nu_{m}\right) E_{k}\left(\nu_{m}\right) \\
M_{k l} & =\sum_{m} U_{m} D_{k}\left(\nu_{m}\right) D_{l}\left(\nu_{m}\right)+V_{m} E_{k}\left(\nu_{m}\right) E_{l}\left(\nu_{m}\right), \\
G_{k} & =D_{k}+i E_{k} .
\end{aligned}
$$

Inversion of the matrix $M$ then gives the coefficients $c_{l}$.

In practice, before using this procedure one first re-defines the function $F$, multiplying it by

$$
\left[\frac{v_{0}^{2}-v^{2}}{v_{1}^{2}}\right]^{-\frac{1}{2} \alpha_{\mathrm{c}}}
$$

so that its $j$-plane branch point is at $j=0 . \chi^{2}$ is a sum over 24 points uniformly randomly distributed over a rectangle in the $\ln (\nu)$ plane:

$$
\nu_{\min }<|\nu|<\nu_{\max }, \quad 0<\operatorname{Arg}(\nu)<\frac{1}{2} \pi .
$$

This is shown in fig. 14. A simple choive of the functions $g_{n}$ and $G_{n}$ is the following:

$$
\begin{aligned}
& g_{n}(j)=\frac{\lambda^{n}}{(n-1) !}(-j)^{n-1} \mathrm{e}^{\lambda j} \\
& G_{n}(\nu)=\lambda^{n}\left(\frac{\nu}{\nu_{1}}\right)\left[\frac{\nu_{0}^{2}-\nu^{2}}{\nu_{1}^{2}}\right]^{-\frac{1}{2}}\left[\lambda+\frac{1}{2} \ln \left[\frac{\nu_{0}^{2}-\nu^{2}}{\nu_{1}^{2}}\right]\right]^{-n} \\
& \simeq\left[1+\lambda^{-1}\left(\ln \frac{\nu}{\nu_{1}}-\frac{1}{2} i \pi\right)\right]^{-n} .
\end{aligned}
$$



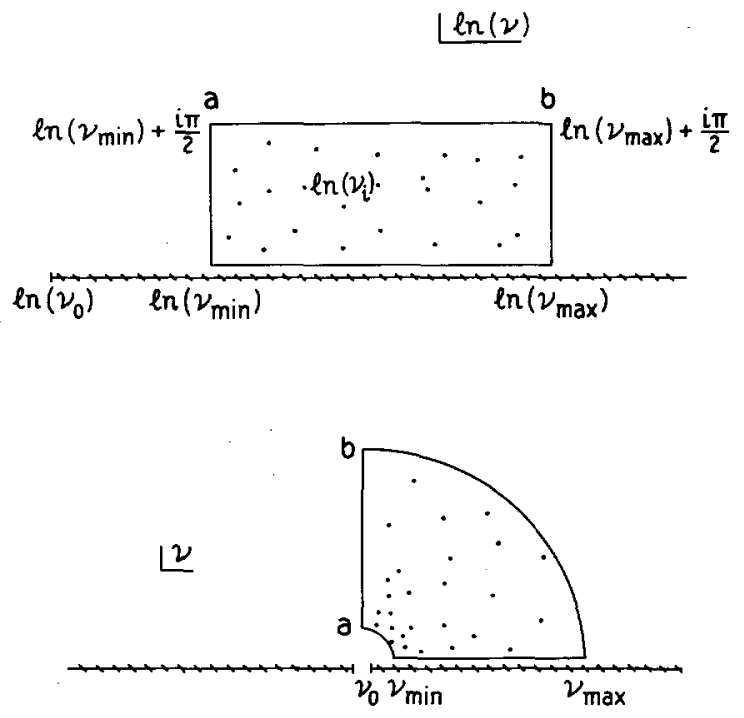

Fig. 14. The region in the complex $\nu$ and $\log \nu$ planes in which randomly distributed points are used to define the $x^{2}$ in eq. (A.3.5). This $x^{2}$ is minimised in determining the $j$-plane cut discontinuity of an absorption model.

However, the procedure which I have been described is essentially the numerical inversion of a Laplace transform, and it is well known that this presents problems. They occur because for most "obvious" choices of the functions $g_{n}$ and $G_{n}$, the ma$\operatorname{trix} M$ is very nearly singular (unless the number of functions, $N$, is very small). The numerical inversion of $M$ introduces large rounding errors which destroy the precision of the answer.

The solution to this problem is to choose a different set of functions $G_{n}^{\prime}$ which are more orthonormal in the region over which $\chi^{2}$ is defined. Then the matrix $M$ becomes more like a unit matrix, and there are no numerical problems when inverting it. Essentially, we replace the $G_{n}$, which are powers of $1 / \log \nu$, by Legendre polynomials in $1 / \log \nu$. The details are as follows:

The $G_{n}$ are imaginary along the upper edge of the rectangle in fig 14 (i.e., $\ln \nu$ $=a+\frac{1}{2} i \pi, \nu=$ pure imaginary). We shall try to find a new set $G_{n}^{\prime}$ which are orthonormal when integrated along the upper edge ( $a \rightarrow b$ in fig. 14). First define a scalar product

$$
\left\langle G_{n} \mid G_{m}\right\rangle=\int_{a}^{b} G_{n}(\nu) G_{m}^{*}(\nu) \mathrm{d}(\ln \nu)=\lambda \int_{y_{1}}^{y_{2}} y^{n-1} y^{m-1} \mathrm{~d} y,
$$




$$
\begin{array}{ll}
y=\left[1+\lambda^{-1} \ln \left(\frac{|\nu|}{\nu_{1}}\right)\right]^{-1}, & y_{1}=\left[1+\lambda^{-1} \ln \left(\frac{\nu_{\max }}{\nu_{1}}\right)\right]^{-1}, \\
y_{2}=\left[1+\lambda^{-1} \ln \left(\frac{\nu_{\min }}{\nu_{1}}\right)\right]^{-1} .
\end{array}
$$

If

$$
z=-1+2\left[\frac{y-y_{1}}{y_{2}-y_{1}}\right],
$$

and the coefficients $d_{j n}$ are defined by

$$
\begin{aligned}
& \sum_{m=1}^{n} d_{m n} y^{m-1}=P_{n-1}(z), \\
& \text { and } \\
& \quad G_{n}^{\prime}=\sum_{i=1}^{n} \sqrt{\frac{2 n-1}{\lambda\left(y_{2}-y_{1}\right)}} d_{i n} G_{i},
\end{aligned}
$$

then

$$
\left\langle G_{n}^{\prime} \mid G_{m}^{\prime}\right\rangle=\delta_{n m}
$$

as required.

In practice I made the following choices: $v_{\min }=3.5 \mathrm{GeV}^{2}, \nu_{\max }=7.5 \times 10^{4} \mathrm{GeV}^{2}$. The $24 \quad \chi^{2}$-defining points had equal weights $U_{i}$ and $V_{i}$ for real and imaginary parts. The function $F(v)$ was expanded in terms of eight $G^{\prime}$ functions, namely:

$$
\begin{array}{lll}
G_{n}^{\prime}(\nu), & n=1,2, \ldots, 6, & \lambda=5, \\
G_{m}^{\prime}(\nu), & m=3,4, & \lambda=1 .
\end{array}
$$

The first six functions parametrize the fine structure of the cut discontinuity near the branch point, and the last two functions are for grosser structure lower in the $j$-plane. With this choice of functions the matrix inversion gives no numerical problems, and the function $F(\nu)$ is typically reproduced accurate to better than one part in $10^{4}$ over the entire fitting range. The resulting cut discontinuity agrees very well with analytic evaluations where these are calculable.

It might be possible to use this technique with real data as input (instead of model amplitudes) to deduce $j$-plane structure in a model-independent way from experimental amplitudes. For instance, at $t=0$ one could use total cross section data, real part data and continuous-moment sum rules to simultaneously constrain the $j$-plane structure. Experience with the method suggests that one needs good data over a wide energy-range before it can be useful. 


\section{Appendix 4. Alternative ways of calculating absorption}

For FESR comparisons I have calculated absorptive cuts by the two-dimensional convolution integral of eq. (A.2.3). If the elastic absorbing amplitude is an exponential in $t$ (such as in eq. (A.2.6)) the convolution can be simplified to a single integral

$$
T_{\mathrm{ABS}}(\nu, t)=\frac{\sigma_{\infty}}{2 \pi} \int_{-\infty}^{0} \mathrm{~d} t^{\prime} \mathrm{e}^{\frac{1}{2} a\left(t+t^{\prime}\right)} I_{n}\left(a \sqrt{t t^{\prime}}\right) T_{\text {pole }}\left(\nu, t^{\prime}\right) .
$$

In this form it is obvious that the absorptive amplitude

(a) Has exact crossing symmetry under $\nu \rightarrow-\nu$ as long as the pole amplitude $T_{\text {pole }}(\nu)$ has exact symmetry under $\nu \rightarrow-\nu$.

(b) Has Regge asymptotic behaviour as $\nu \rightarrow \infty$ in any complex direction.

(c) Has the form of a single $j$-plane cut, stretching from $j=\alpha_{\mathrm{c}}=\alpha_{\text {pole }}(0)$ to $j=-\infty$, without any daughter cuts (at $\left.\alpha_{\mathrm{c}}=\alpha_{\text {pole }}(0)-n, n=1,2 \ldots\right)$.

Properties (a) and (b) are essential prerequisites for an FESR comparison; it can be argued that they are essential to any dual amplitude. Property (c) was desirable, to minimise the effect of low-lying singularities on the FESR, in accord with the usual interpretation of duality.

There are other ways of calculating absorption, which are equivalent to the above form for large positive $s$, but which extrapolate differently to low energies. If one could find such a form which has a sensible motivation, which has properties (a) and (b), and which gives a better extrapolation to low energies than the previous form, then one would have to reconsider the statement of duality in terms of leading $j$ plane singularities (since the new form would only differ from the old by having larger non-leading $j$-plane singularities). This appendix records some (unsuccessful) attempts to find such a form of absorption.

The integral (A.4.1) diverges for some small $\nu$ value, producing a pole in the amplitude. (This pole is usually well inside the FESR contour, so could easily be shifted slightly to hide it in the physical region cuts, without affecting the semi-circle integral significantly. It is not relevant to FESR comparisons, since all Regge models contain some locally unrealistic low-energy structure; in the real world this structure is presumably corrected by low-lying $j$-plane singularities which are small in the semicircle integral.) One might hope, by removing this pole, to get an amplitude with a better low-energy extrapolation. There are several possible ways of removing the pole:

(i) Subtract it explicitly. This introduces a series of low-lying right-signature fixed poles in the amplitude, and is therefore not an attractive solution.

(ii) Truncate the cut integral (eq. (A.4.1)) at some constant negative t-value. In other words, replace $\int_{-\infty}^{0} \mathrm{~d} t^{\prime}$ by $\int_{t_{\mathrm{c}}}^{0}$. This makes the cut discontinuity vanish for all $\alpha<\alpha_{\rho}\left(t_{\mathrm{c}}\right)$, leaving it unchanged above this $\alpha$ value. However, the $j$-plane analysis of sect. 4 shows that such a solution, by removing the low-lying cut discontinuity, is not possible. Essentially all parts of the cut which contribute appreciably to the 
FESR, also contribute importantly in the $6 \mathrm{GeV}$ amplitudes - one cannot improve the FESR agreement without spoiling the agreement in the high energy amplitudes.

(iii) Truncate the cut integral at $\theta_{\mathrm{cm}}^{\mathrm{s}}=90^{\circ}$. In the equal mass case, replace $\int_{-\infty}^{0} \mathrm{~d} t^{\prime}$ by $\int_{\frac{1}{2}\left(4 m^{2}-s\right)}^{0} \mathrm{~d} t^{\prime}$. This gives an amplitude which differs from the original form by a calculable function $V(\nu, t)$. It is easy to see that $V$ vanishes exponentially as $\nu \rightarrow+\infty$; the two forms do not differ by daughters, but differ by a non-power behaved function. For negative $\nu$-values, the modified form is an integral over positive $t$-values; it grows exponentially as $\nu \rightarrow-\infty$. By this modification, therefore, one loses both $s-u$ crossing symmetry and Regge behaviour, two prerequisites of a dual amplitude. Simple variations on this theme can be shown to give the same problems.

(iv) Absorb in partial waves rather than impact parameter amplitudes. Partial wave amplitudes are defined by an integral in the physical region, $0>t>4 m^{2}-s$. So again the absorbed amplitude is given by an integral, with limits $t=0$ and $t=4 m^{2}-s$, of the pole amplitude. For negative $\nu$ this is again an integral over positive $t$ values, and the amplitude diverges exponentially. While perhaps improving the behaviour of the amplitude in the region of small positive $\nu$, we have destroyed its crossing symmetry and Regge behaviour for negative $\nu$-values.

\section{References}

[1] G.C. Fox in Phemenology in particle physics (Caltech, 1971);

R.J.N. Phillips in Proc. of Amsterdam Int. Conf. on elementary particles (Nor th-Holland, Amsterdam, 1971);

C. Michael in Proc. of the 4th Int. Stony Brook Conf. on high energy physics, Oxford, 1972.

[2] M. Ross, F.S. Henyey and G.L. Kane, Nucl. Phys. B32 (1971) 429.

[3] M. Fukugita, University of Tokyo preprint (November, 1972);

M. Fukugita and T. Inami, Nucl. Phys. B44 (1972) 490;

C. Schmid, in Phenomenology in particle physics (Caltech, 1971).

[4] F. Halzen and C. Michael, Phys. Letters 36B (1971) 110 ;

G. Cozzika et al., Phys. Letters 40B (1972) 281;

P. Johnson et al., Phys. Rev. Letters 30 (1972) 242.

[5] A.C. Irving, A.D. Martin and V. Barger, CERN preprint TH1585 (1972);

C.B. Chiu and E. Ugaz, Phys. Letters 43B (1973) 327.

[6] B.J. Hartley and G.L. Kane, Phys. Letters 39B (1972) 531; University of Michigan preprint (January, 1973).

[7] G.A. Ringland, R.G. Roberts, D.P. Roy and Tran Thanh Van, Nucl. Phys. B44 (1972) 395.

[8] R. Hong Tuan, G. Kaplan and G. Sanguinetti, Nucl. Phys. B32 (1971) 655;

P.D.B. Collins and R.A. Swetman, University of Durham preprint (1972).

[9] G. Girardi et al., Nucl. Phys. B47 (1972) 445.

[10] A. Martin and P.R. Stevens, Phys. Rev. D5 (1972) 147; Michigan State University preprint (1972);

J.W. Coleman and R.C. Johnson, Phys. Rev. D, to appear;

M. Ross, Phys. Letters 38B (1972) 321;

J. Anderson and K.J.M. Moriarty, Royal Holloway preprint RHCM $71 / 4$ (1972). 
[11] F. Henyey, G. Kane, J. Pumplin and M. Ross, Phys. Rev. 182 (1969) 1579.

[12] M. Jacob, CERN Th. 1010 and Proc. of the Lund Int. Conf., 1969;

R.J.N. Phillips and D.P. Roy, to be published;

C. Schmid, in Proc. of the Amsterdam Int. Conf. on high energy physics, 1971 (North Holland, Amsterdam, 1972).

[13] D. Sivers and J. Yellin, Ann. of Phys. 55 (1969) 107.

[14] R.C. Johnson, Phys. Rev. D5 (1972) 2768.

[15] S. Almehed and C. Lovelace, Nucl. Phys. B40 (1972) 157.

[16] R. Ayed et al., Saclay preprint (1972).

[17] R.P. Worden, Phys. Letters 40B (1971) 260.

[18] G.V. Dass, C. Michael and R.J.N. Phillips, Nucl. Phys. B9 (1969) 549;

F. Halzen, private communication.

[19] V. Barger and R.J.N. Phillips, Phys. Rev. 187 (1969) 2210.

[20] H. Hogaasen and C. Michael, Nucl. Phys. B44 (1972) 214.

[21] C. Lovelace, in Proc. of the Batavia Int. Conf. on high energy physics, 1972;

E.L. Berger and D. Sivers, Argonne preprint (1972).

[22] R.D. Field, Ph. D. thesis; Lawrence Berkeley preprint; Phys. Rev. D5 (1972) 86.

[23] R.C. Johnson, Phys. Letters 38B (1972) 325.

[24] V. Barger, K. Geer and F. Halzen, Nucl. Phys. B44 (1972) 475; C. Schmid, ref. [3].

[25] D. Hill et al., Phys. Rev. Letters 30 (1972) 239.

[26] J.S. Ball, G. Marchesini and F. Zachariasen, Phys. Letters 31B (1970) 583; D.P. Roy in Proc. of the 6th Rencontre de Moriond, 1971.

[27] R.P. Worden, Nucl. Phys. B37 (1972) 253.

[28] F. Elvekjaer, T. Inami and G.A. Ringland, RHEL preprint, to appear.

[29] H. Harari, Phys. Rev. Letters 26 (1971) 1400; Ann. of Phys. 63 (1971) 432; G.I. Ghandour and R.G. Moorhouse, Berkeley preprint (December, 1971); M.F. Coirier et al., Nucl. Phys. B44 (1972) 157.

[30] J. Alcock and N. Cottingham, Nucl. Phys. B31 (1971) 443; Univ. of Bristol preprint (1972).

[31] F. Elvekjaer and E. Pietarinen, Nucl. Phys. B45 (1972) 621;

F. Elvekjaer, RHEL preprint RPP/T/36 (1972);

G. Ross, Nucl. Phys. B31 (1971) 113; B41 (1972) 272.

[32] C. Michael, Nucl. Phys. B8 (1968) 431.

[33] V. Singh, Phys. Rev. 129 (1969) 1889. 